\title{
Sustainable Nanomaterials: A Greener Future Avenue?
}

\section{Rafael Luque*}

\author{
Department of Organic Chemistry, University of Cordoba, Spain
}

*Corresponding author: Rafael Luque, Professor, Department of organic chemistry, University of Cordoba, Cordoba, Spain, E-mail: q62alsor@uco.es

Citation: Rafael Luque (2013) Sustainable Nanomaterials: A Greener Future Avenue? J Mater Sci Nanotechnol 1(1): e106. doi: 10.15744/2348-9812.1.e106

\section{Received Date: June 23, 2013 Accepted Date: July 29, 2013 Published Date: August 01, 2013}

The field of nanoscience has experienced a staggering number of advances in recent years with regards to a wide range of disciplines including physics, chemistry, materials science, biology and medicine [1]. Although most of these are still closely related to laboratory practices and yet far from entrepreneurial activities, many of these nanomaterials are currently commercially available (e.g. silica nanowires, silicon wafers, carbon nanotubes, graphene and derivatives) and have paved the way to enormous developments in the preparation of functional nanomaterials for various applications [1,2]. Generally speaking, environment and sustainable considerations around the synthetic protocols currently in place for the fabrication of nanoentities are scarce and nanosafety procedures in terms of toxicity, handling of nanomaterials and their environmental impact are still not yet sufficiently developed (in the available cases). Considering the synthesis of some of these nanomaterials, protocols often involve high temperatures and pressures, the use of hazardous reagents and/or solvents or include little details to be accounted for in terms of environmental considerations (e.g. environmental impact, green metrics, handling risks, toxicity). Taking into account the essentially different nature and properties (often unknown) of such nanoentities, an increasing number of restrictions, handling practices and safety procedures are currently present in several laboratories and working places in which nanomaterials are handled on a daily basis $[3,4]$. A complete set of new and updated information on characteristics and properties (as well as risks and toxicity issues in their manipulation) is truly needed to better understand and establish the most acceptable working practices for nanotechnologies. However, a benign by design concept should be promoted in all nano-related practices, aiming to target the development of future sustainable nanoprotocols and environmentally compatible nanomaterials $[5,6]$.

This editorial contribution advocates for more thoughtful and carefully design methodologies that take into account environmentally sound protocols for nanomaterials development. These should range from low temperature ambient pressure methods, avoidance of hazardous chemicals, solvent-free protocols to alternative greener technologies such as mechanochemistry-ball milling-[7], microwave irradiation $[2,8,9]$ and sonication [10] as well as life cycle and risks assessments (whenever possible and appropriate) [11] to analyse and considerate toxicity, risks and environmental impact of the synthesis, handling and utilization of nanomaterials in our daily practices in view of a future implementation of nanotechnologies in our future society [12].

In any case, judging from recently developments in the field, the era of environmentally compatible nanomaterials for future sustainable applications is envisaged to be part of a more sustainable and economically competitive society which this author particularly hopes to enjoy as a reality within the next 30 years.

\section{Acknowledgement}

Rafael Luque gratefully acknowledges support from the Spanish MICINN via the concession of a RyC contract (ref. RYC 2009-04199) and funding under projects P10-FQM-6711 (Consejeria de Ciencia e Innovacion, Junta de Andalucia) and CTQ2011 28954-C02-02 (MICINN).

\section{References}

1. Ozin GA, Arsenault AC, Cademartiri L (2008) Nanochemistry: A Chemical Approach to Nanomaterials. (2nd edn), Royal Society of Chemistry, Cambridge, UK.

2. Rao CNR, Müller A, Cheetham AK (2006) The Chemistry of Nanomaterials: Synthesis, Properties and Applications, Wiley VCH, Weinheim, New Jersey, USA.

3. Nanosafety cluster

4. Nanosafety at the OECD. The first five years 2006-2010.

5. Luque R, Varma RS (2012) Sustainable preparation of nanoparticles: Methods and Applications. Royal Society of Chemistry, Cambridge, UK.

6. Murphy CJ (2008) Sustainability as an emerging design criterion in nanoparticle synthesis and applications. J Mater Chem 18: 2173-2176.

7. James SL, Adams CJ, Bolm C, Braga D, Collier P, et al. (2012) Mechanochemistry: opportunities for new and cleaner synthesis. Chem Soc Rev 41: 413-447.

8. Pineda A, Balu AM, Campelo JM, Romero AA, Carmona D, et al. (2011) A dry milling approach for the synthesis of highly active nanoparticles supported on porous materials. ChemSusChem 4: 1561-1565.

9. Horikoshi S, Serpone N (2013) Microwaves in nanoparticle synthesis, Fundamentals and Applications. Wiley-VCH, New Jersey, USA. 
10. Manoharan SS, Rao ML (2004) Sonochemical Synthesis of Nanomaterials. Encycl Nanosci Nanotechnol 10:67-82.

12. Balas F, Arruebo M, Urrutia J, Santamaria J (2010) Reported nanosafety practices in research laboratories worldwide. Nat Nanotechnol 5: 93-96.

11. Stella GM (2011) Carbon nanotubes and pleural damage: perspectives of nanosafety in the light of asbestos experience. Biointerphases 6: 1-17.

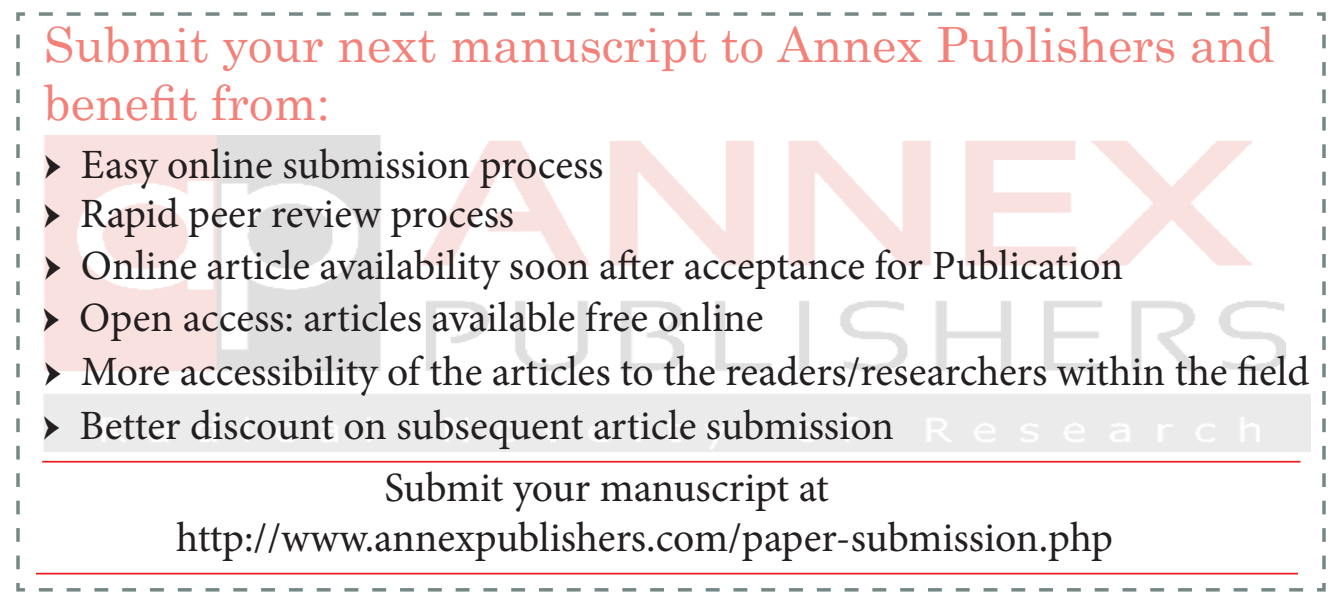

